\title{
A COMPARISON OF ASTROMAG COILS MADE WITH ALUMINUM AND COPPER BASED SUPERCONDUCTOR
}

\author{
M. A. Green \\ Lawrence Berkeley Laboratory \\ University of California \\ Berkeley, CA 94720
}

1990 Applied Superconductivity Conference

Snowmass, CO

September 24-28, 1990

*This work was supported by the Office of Astrophysics, NASA, and the Office of Basic Energy Science, U.S. Dept. of Energy, under Contract No. DE-AC03-76SF00098. 


\title{
A COMPARISON OF ASTROMAG COILS MADE WTTH ALUMINUM
} AND COPPER BASED SUPERCONDUCTOR

\author{
M. A. Green \\ Lawrence Berkeley Laboratory \\ University of Califomia \\ Berkeley, CA 94720
}

\begin{abstract}
The use of aluminum matrix superconductor in the coils for the ASTROMAG magnet will increase the integrated field for doing particle astrophysics in space as compared to equal mass coils made with copper matrix superconductor. The increased ability to detect charged particles can be achieved without decreasing the current margin of the superconductor in the coils. The use of a low resistivity aluminum matrix conductor increases the energy need to initiate a quench by two orders of magnitude. The current decay time constant during a quench is substantially increased. As a result, the quench energy dumped into the helium tank is reduced (The ASTROMAG coils are thermally decoupled from the helium tank.), and the forces on the shields and shells due to eddy currents will be lower. This paper also describes the problems associated with the use of aluminum matrix superconductor in the coils.
\end{abstract}

\section{Background}

The ASTROMAG' superconducting magnet consists of two supercunducting coils powered with opposite polarity so that the net magnetic dipole moment is zero. The two coils, which are two meters apari, are attached ro a 3440 liter superfluid helium tank which supplies cooling for the coils for up to four years in space.(See Fig. I for a basic configuration of the ASTROMAG magnet coils mounted on the superfluid helium tank.)

There are two particle astrophysics experiments, each mounied on an end of the cryosiat. The closest physics detector is located 12 to 15 centimeters from the superconducting magnet coil for that experiment. The heavy nuclei experiment requires that $\mathrm{C}_{1}=$ maximize the field integral through the detector. In order to maximize the field integral, one must increase the ampere turns in the coils. The magnet stored energy goes up as the ampere tums squared for a given coil package. The mass of the coils and the cryogenic system goes up as the stored energy.

The baseline ASTROMAG superconducting coil at the end of phase $A$ used a copper matrix superconductor to generate the magnetic field ${ }^{2}$. The copper based coil cross-section is shown in Fig. 2. The copper baseline magnet parameters are shown in the table below Fig. 2. Each copper based coil will carry $1.98 \mathrm{MA}$ turns and has a mass of about $325 \mathrm{~kg}$. The copper based superconducior will operate at 85 to 90 percent of critical current along the load line when the coil temperature is $4.2 \mathrm{~K}$. The magnet stored energy at the design current is $11 \mathrm{MJ}$.

\footnotetext{
This work was supported by the Office of Astrophysics, NASA, and the Office of Basic Energy Science, U.S. Lept. of Energy, under Contract No. DE-AC03.76SF00098.
}

\section{Aluminum Marrix Coil Versus Copper Marrix Coils}

Since weight is always an imporzant factor in any space mission, the use of aluminum matrix superconductor in place of copper matrix conductor has been studied. Studies made in May of $1989^{3}$ showed that the number of ampere turns in the coil could be increased without increasing the magnet weight by using superconductor with an aluminum matrix. A number of other advantages also became apparent.

The advantages of aluminum matrix superconducting coils over copper matrix superconducting coils for the ASTROMAG magnet design are as follows: 4

1. For a given coil mass (including the coil support structure), the aluminum matrix superconducting coil will carry more current. This means that the maximum detectable momentum can be increased in the experiments without altering the particle detoctors.

2. The energy needed to initiate a quench in the aluminum matrix superconductor in the coil is about two orders of magnitude higher than it is for the comparable copper matrix superconducting coil.

3. The quench decay time for the aluminum matrix coil will be about a factor of two longer than for the comparable copper matrix coil. As a result, less energy will be dumped into the helium tank during a quench and the forces on the shields and shells due to eddy currents will be lower.

4. The energy density for the aluminum based superconducting coil is higher than it is for the copper based superconducting coil.

The two potential problems which are associated with the use of the aluminum masrix superconductor in the ASTROMAG coils are as follows:

1. The aluminum matrix coils are strain limited because the very pure aluminum matrix material is not capable of carrying large forces without yielding.

2. Superconductor with a pure aluminum matrix has very different quench propagation characteristics from copper based superconductor. Quench protection is more of a problem at intermediate currents than it is at higher currents.

The proposed aluminum matrix superconductor coil cross-section is shown in Fig. 3. The parameters for the aluminum matrix coil are shown in the table atrached to Fig. 3. 


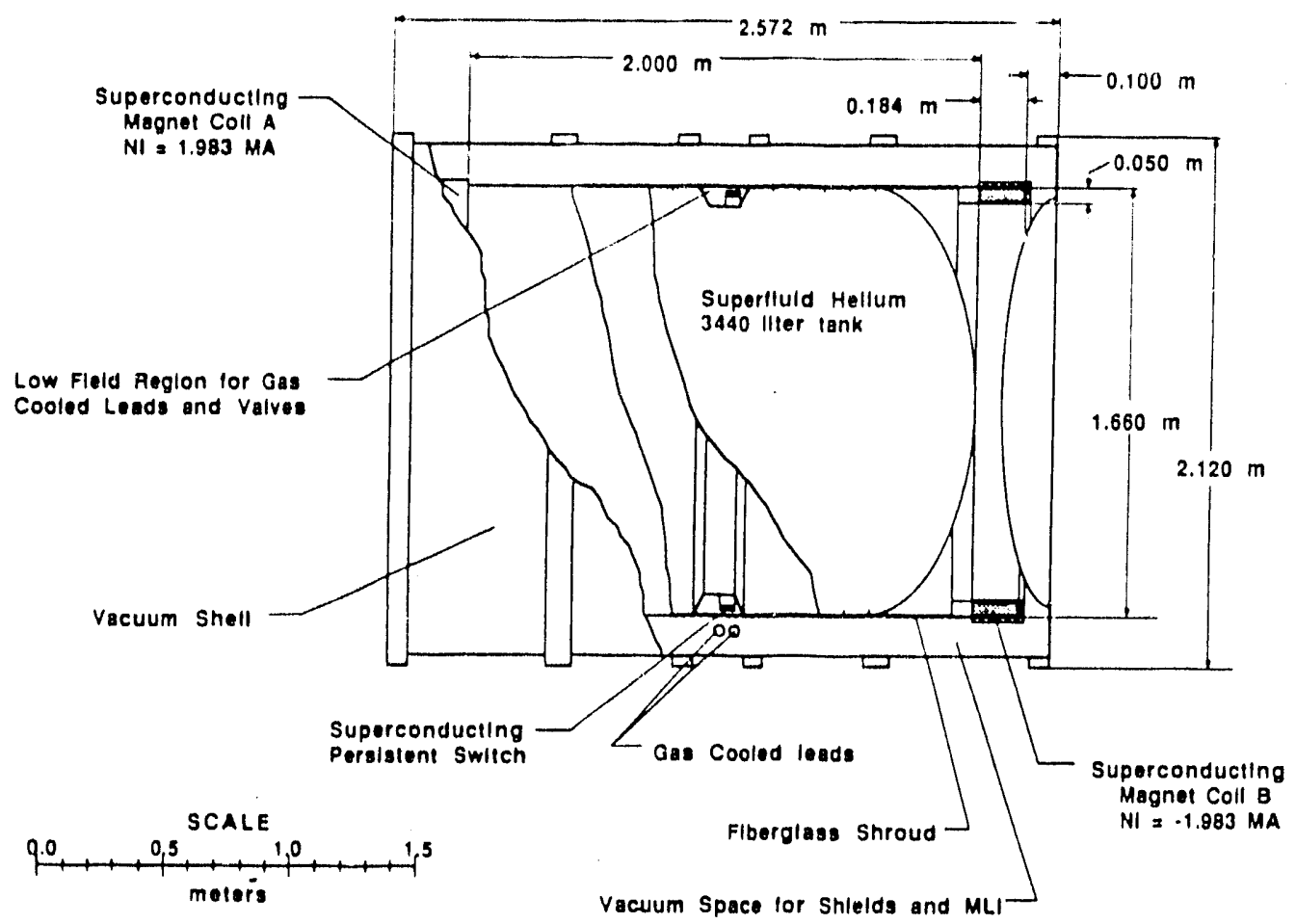

Fig. 1. The Baseline Magnet and Cryostat Configuration for ASTROMAG

With Copper Matrix Superconducting Coils.

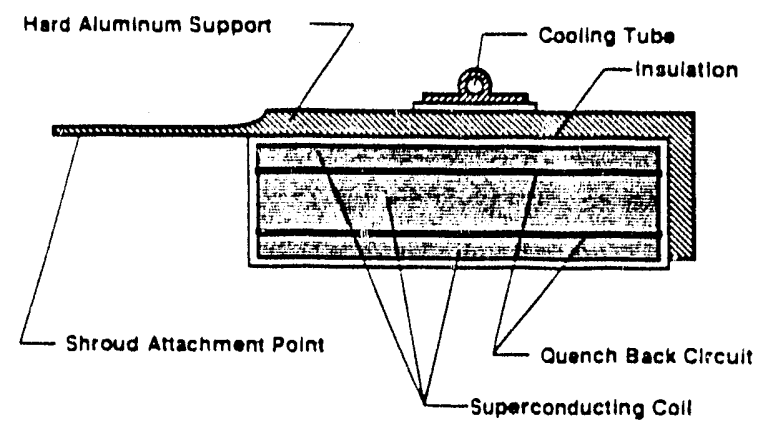

COPPER MAGNET PARAMETERS

\begin{tabular}{|c|c|}
\hline 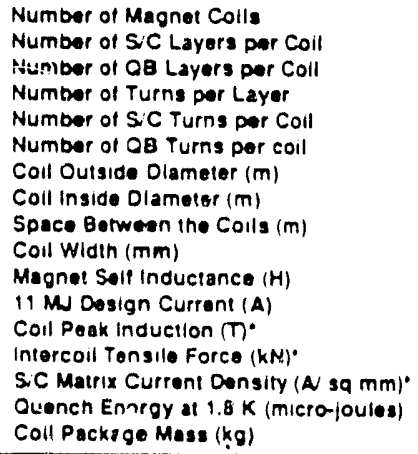 & $\begin{array}{r}2 \\
34 \\
4 \\
72 \\
2448 \\
288 \\
1.66 \\
1.56 \\
2.00 \\
184.00 \\
33.52 \\
810.09 \\
6.74 \\
2514 \\
405 \\
9.6 \\
322.7\end{array}$ \\
\hline
\end{tabular}

- At the $11 \mathrm{~mJ}$ Design Coil Current

- 25.6 intric cons

Fig. 2. A Cross-Section of the Copper Matrix Superconducting Coil for ASTROMAG.

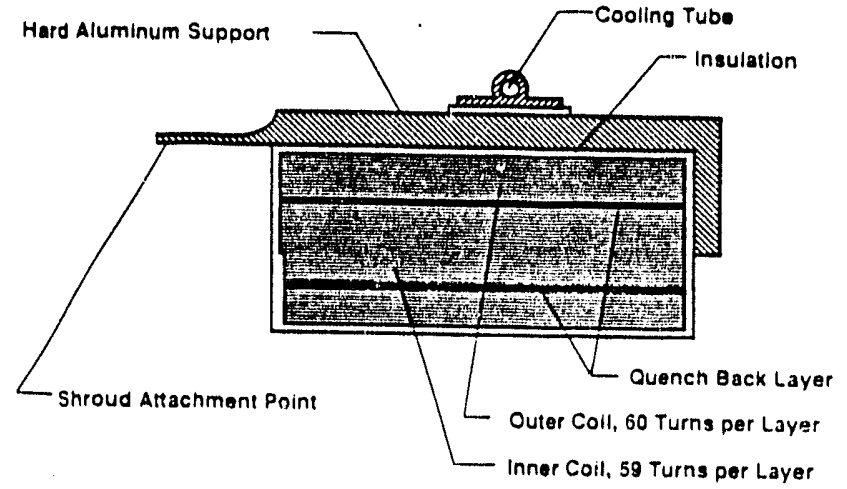

ALUMINUM MAGNET PARAMETERS

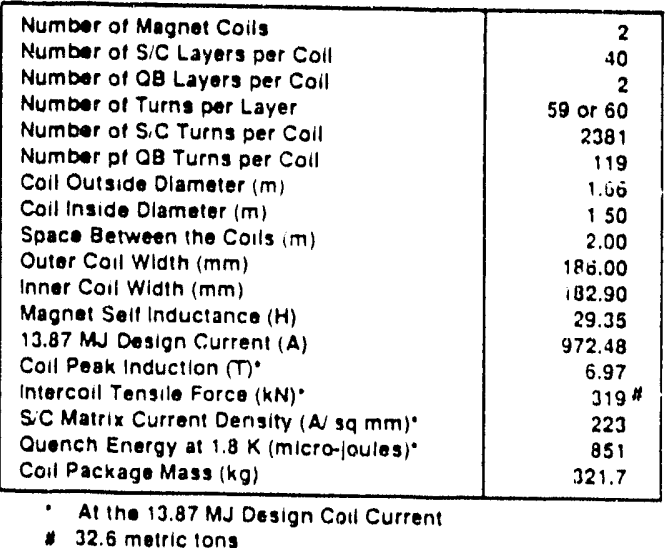

Fig. 3. A Cross-section of an Aluminum Matrix Superconducting Coil for ASTROMAG Without the Use of Advanced Composites 
Srain Within The Aluminum Marix Superonductor and the Coil Package

When one looks at the stress and strain within the ASTROMAG aluminum matrix superconducting coil, one must look at the properties of the coil materials. The basic aluminum matrix superconducting coil package shown in Fig. 3 has a total crosssectional area of 148.8 square centimeters. The coil shown in Fig. 3 has 2381 turns of aluminum matrix superconductor which has a cross-sectional area of 4.3 square millimeters per turn. (The copper matrix superconductor used in the coil shown in Fig. 2 has a crosssectional area of 2.0 square millimeters per tum.) Of the 148.8 square centimeters of coil cross-sectional area, about 108 square centimeters is aluminum matrix superconductor. The remainder of the cross-section is fiberglass epoxy insulation. The proposed superconductor for the coil consists of 1 part Nb-Ti, 0.8 parts copper, and 4 parts $R R R=1000$ pure aluminum.

The aluminum matrix coil is strain limited. One wants to limit the coil average strain to less than $0.3 \%$ in order to avoid coil training. ${ }^{5}$ It is proposed that the aluminum matrix coil shown in Fig. 3 consist of potted coil, shrink fitted into a hard aluminum support ring. The shrink fitted coil will be squeezed by the support ring as the coil is cooled down. As the coil is powered, the hoop forces in the coil can be partially taken up by the support ring without passing this force across a tensile or shear joint. As a result, an important cause of training is eliminated. It is proposed that the coil could be wound and vacuum impregnated on a retractable winding mandrel. The finished potted coil is then machined to an exact dimension so that is can be shrink fitted into a machined support ring. The coil will be cooled by helium flowing through a tube installed onto the outside of the support ring. The cooling tube should be insulated from the support ring in order to control the pressure build up during a quench of the magnel.

The step in the coil shown in Fig. 3 is intentional. The outer 22 layers have 60 turns while the inner 20 layers only have 59 turns. This moves the center of the intercoil force radially outward. As a result, the mechanical moment at the shroud attachment point is reduced by over a factor of two.

\section{Quench Protection of Aluminum Matrix Superconducring Coils}

Aluminum matrix superconductor in the ASTROMAG coils poses questions of safety during a magnet quench. The questions posed are: 1) Will the magnet quench without the superconductor melting or the insulation burning at the place in the coil where the quench was initiated? (This is usually a high field point or some kind of slip point in the coil.) 2) Can both of the ASTROMAG coils be driven normal during the quench process? 3) Will the persistent switch be protected should it become normal while the gas cooled electrical leads are detached? The answer to all three questions is yes provided proper design steps are taken to insure fail safe quenching of the coil.

Fail safe quench protection at full design magnet current appears to be possible without using quench back. ${ }^{6}$ Quench back frotn the support ring will insure that the second coil will be normal along with the first when one coil or the persistent switch quenches. Quench back from the support ring does not appear to be adequate at currents 50 to $70 \%$ of full current. Quench propagation along the wire is very fast at high currents, but much slower as the curren goes down. ${ }^{7}$ Transverse quench velocities scale with the quench propagation velocity along the wire.

If a pure aluminum quench back circuit is installed within the coil as shown in Fig. 3, the entire coil appears to go normal fast enough to insure fail safe quenching over the full range of potential operating currents, despite the fact that quench propagation velocities are much lower at low currents. The addition of a pure aluminum quench back circuit reduces the total ampere turns in the coil by about 2 percent for a given operating margin. The pure aluminum quench back circuit is a hindrance unless the magnet charge time is long. This hindrance can be alleviated by putting cold diodes across the quench back circuit (see Fig. 4).

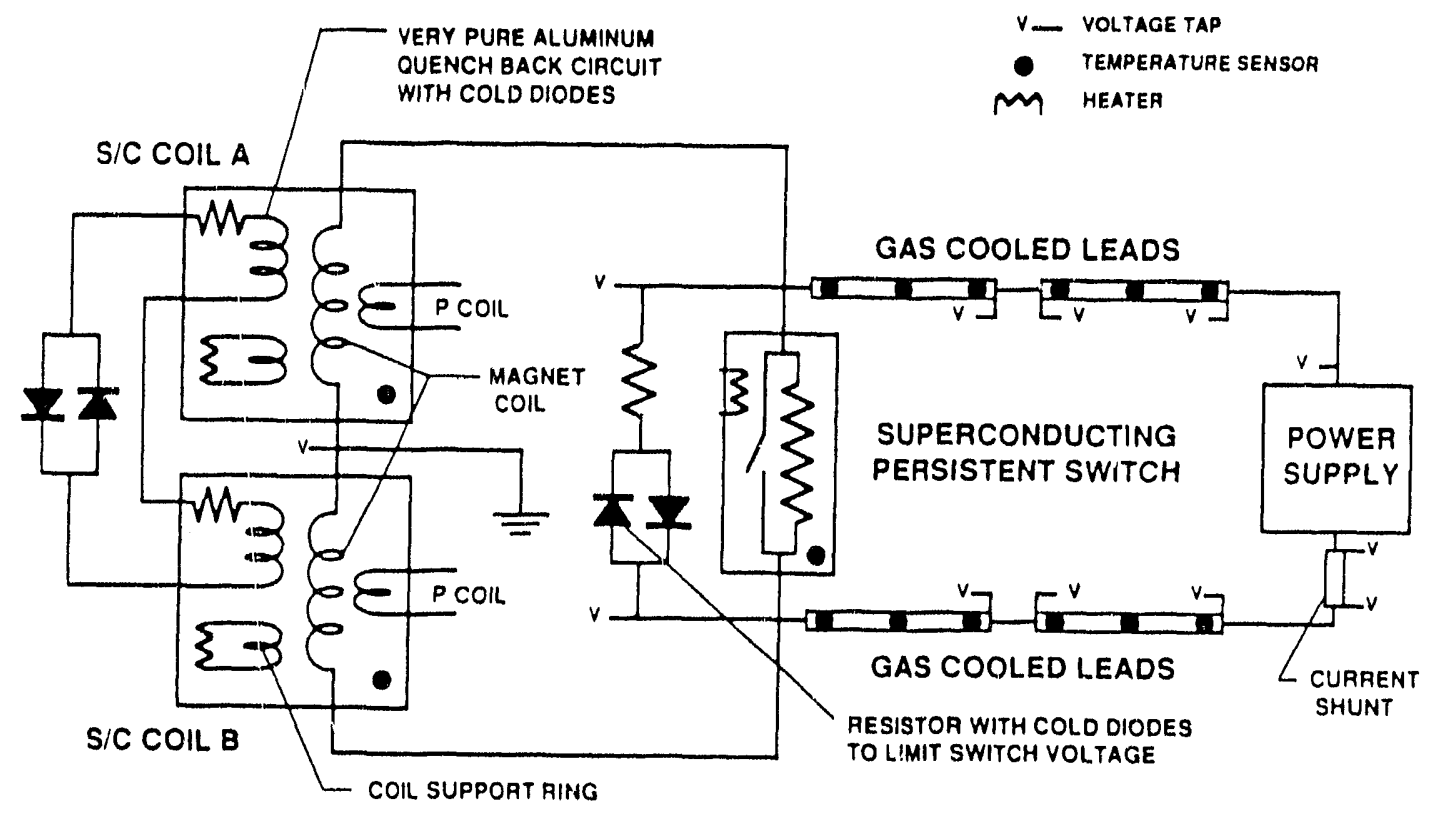

Fig. 4. The Electrical Circuit Diagram for the ASTROMAG Magnet System With Quench Back Circuits and Cold Diodes.

$$
-3-
$$


Improvement of Coil Performance through the

Use of idvanced Composite Materials

A change of the conductor from a copper matrix superconductor to an aluminum matrix superconductor increases the maximum detectable momentum (MDM) of the experiments by 15 percent. (To first order, MDM is proportional to the product of ampere turns and average coil diameter.) Aluminum matrix superconducting coils are strain limited. In order to increase the number of ampere turns for a given magnet coil mass, one has to increase the amount of superconuctor per turn and one must provide additional material which is capable of carrying the magnetic forces.

The most effective way of adding strength carrying material to the coil package is to use material which have a nigh elastic modulus as well as high strength. The added material must also have a low density. This eliminates metals such as the stainless steels and materials such as glass and Kevlar fibers. Also eliminated are most of the light metals. (Most do not have a high enough modulus of elasticity.) Advance composites such as carbon fiber and boron fiber appear to be good candidates if they are used in the uniaxial form in the hoop direction 8 .

Figure 5 shows an ASTROMAG coil cross-section which uses unidirectional boron fiber in both the superconductor and the insulation layers between the conductor layers. The superconductor propused for the coil shown in figure 5 would consist of 1 part $\mathrm{Nb}$ Ti, (). 8 parts copper, 0.5 parts uniaxial boron aluminum fiber composite (about 50 percent boron), and 3 parts RRR $=1000$ pure aluminum. The interlayer insulation would consist of boron-epnxy tape between layers of Kapton. The coil shown in figure 5 has no hard aluminum structure outside of the coil. A relatively thin 6061 aluminum structure is used to carry the longitudinal inter-coil force to the shroud. The coil shown in figure 5 will permit the MDM in the experiment to go up 20 percent compared to the coil shown in figure 3.

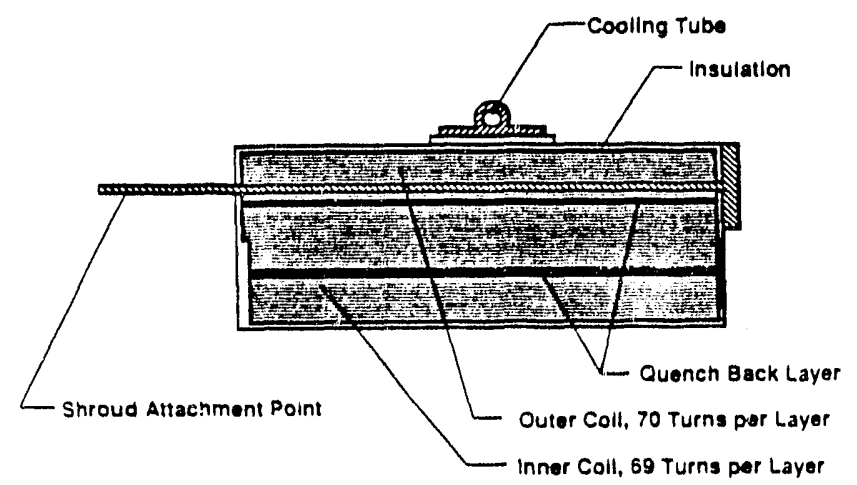

ALUMINUM MAGNET PARAMETERS

\begin{tabular}{|c|c|}
\hline 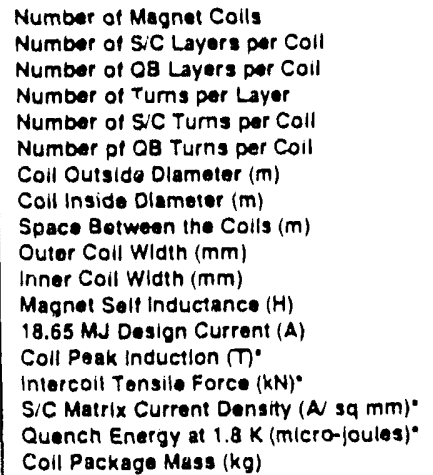 & $\begin{array}{r}2 \\
38 \\
2 \\
69 \text { or } 70 \\
2641 \\
139 \\
1.74 \\
1.59 \\
2.00 \\
217.00 \\
213.90 \\
37.30 \\
1000.0 \\
7.10 \\
468 \\
230 \\
528 \\
320.8\end{array}$ \\
\hline
\end{tabular}

- At the 18.65 Mu Desion Coll Current

- $\$ 7.8$ metric ton

Fig. 5. A Cross-Section of an Aluminum Matrix Superconducting Coil for ASTROMAG Which Uses Uniaxial Boron Composites.

\section{Summary}

An ASTROMAG coil set which uses aluminum matrix superconductor is both possible and desirable. Coils made from aluminum matrix superconductor carry more current per unit mass. they require more energy in order to initiate a quench, and they decay at a slower rate during a quench. The aluminium matrix coils can be made so that they are capable of carrying both the longitudinal and hoop stresses. Quench protection of the aluminum matrix coils appears to be not much different than for a copper matrix superconducting coil system. The quench protection method studied will protect both coils and the persistent switch. The use of advanced composites along with the aluminum matrix superconductor in the ASTROMAG magnet can increase the MDM of the experiment by 38 percent without a change in the magnet coil mass.

\section{References}

1. M. A. Green, et al., IEEE Transactions on Magnetics MAG. 23, No. 2, 1240, 1987.

2. ASTROMAG Phase-A Study Final Report, prepared by Advanced Missions and Analysis Office, Code 402, NASA-GSFC, Greenbelt, MD 20771, 21 December 1989.

3. M. A. Green, "A Comparison of Aluminum Matrix Coils for ASTROMAG With The Copper Matrix Coils," Lawrence Berkeley Laboratory LBID-1506, May 1989.

4. M. A. Green, "The Consequences of Changing the ASTROMAG Baseline Magnet Design to a Design Which Uses an Aluminum Matrix Superconductor,".Lawrence Berkeley Laboratory LBID-1598, April 1990.

5. J. W. Ekin, et al., Nonmetallic Materials and Composites at Low Temperatures, p301, Plenum Press, New York, (1979).

6. M. A. Green, Cryogenics 24, 659, 1984

7. M. Scherer and P. Turowski, Cryogenics 18, 515, 1978

8. S. W. Tsai and H. T. Hahn, Introduction to Composite Materials, Technomic Publishing Co. Inc., Lancaster, (1980) 

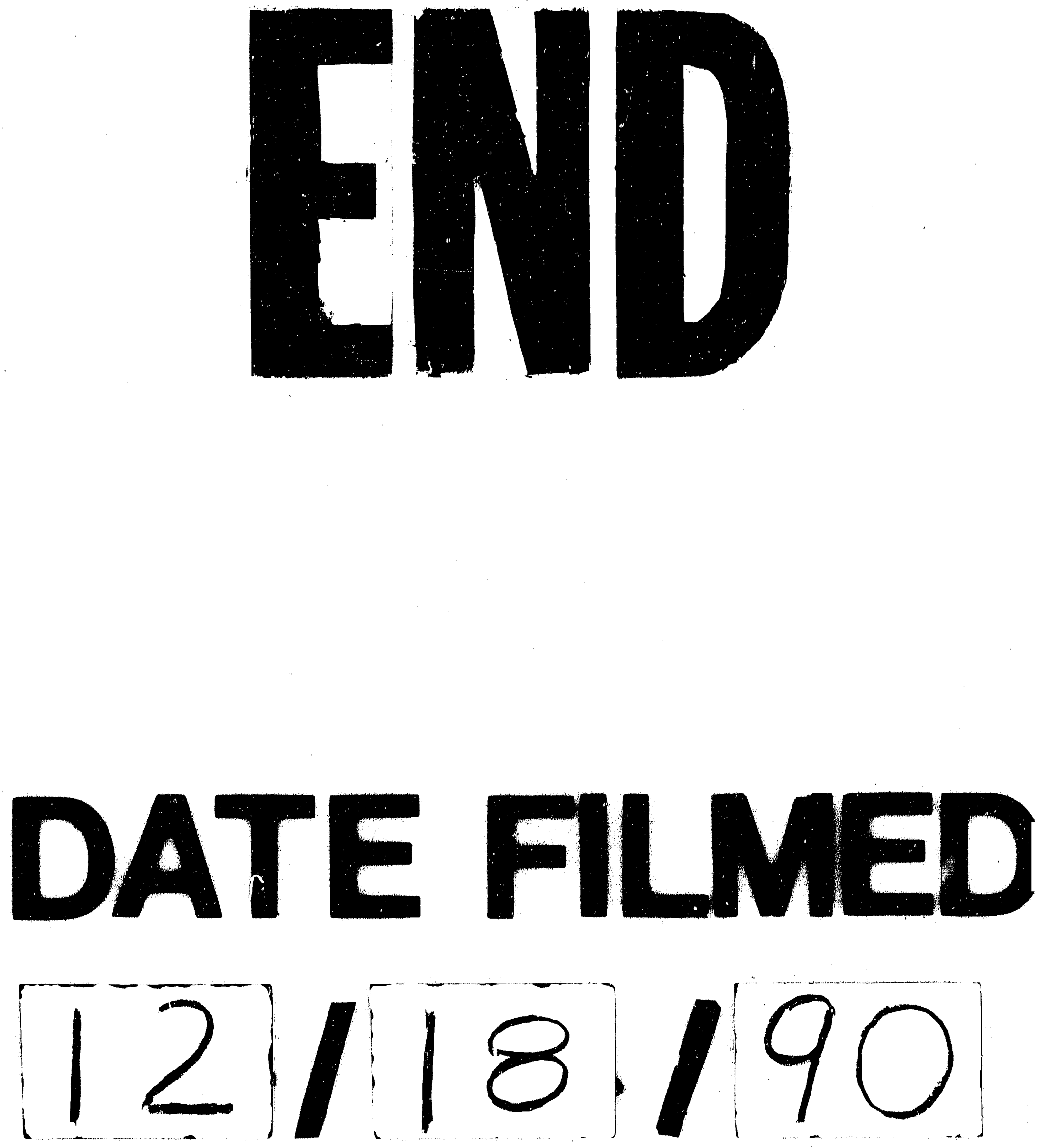
